\title{
ONCHOCERCIASIS CONTROL: BIOLOGICAL RESEARCH IS STILL NEEDED
}

\author{
BOUSSINESQ M.*
}

\section{Summary:}

Achievements obtained by the onchocerciasis control programmes should not lead to a relaxation in the biological research on Onchocerca volvulus. Issues such as the Loa loa-related post ivermectin serious adverse events, the uncertainties as to whether onchocerciasis can be eliminated by ivermectin treatments, and the possible emergence of ivermectin-resistant $O$. volvulus populations should be addressed proactively. Doxycycline, moxidectin and emodepside appear to be promising as alternative drugs against onchocerciasis but support to researches in immunology and genomics should also be increased to develop new control tools, including both vaccines and macrofilaricidal drugs.

KEY WORDS : onchocerciasis, Onchocerca volvulus, treatment, ivermectin, adverse reactions, elimination, resistance, genomics, vaccine.

M ore than 60 million treatments with ivermectin (IVM) are now distributed every year against onchocerciasis. These treatments have had a marked impact on the disease, and it is estimated that in 2005 the prevalence of onchocercal blindness in the countries included in the African Programme for Onchocerciasis Control (APOC) was reduced by $23 \%$ when compared with the pre-treatment figure (Stolk et al., 2007). This has been made possible by the existence of a "wonder drug" donated by Merck \& Co., the development of an innovative community-directed strategy which is now regarded as a model for delivering other health interventions, and a unique partnership between the endemic countries, international and non-governmental organizations, and Merck \& Co. Due to these successes, onchocerciasis is now certainly less "neglected" than other tropical diseases. However, it might become so if some problems the control activities do have to face are occulted. This paper summarizes some of these issues, as well as the biological researches to be pursued so that the gains of the control programmes can be maintained.

\footnotetext{
* Institut de Recherche pour le Développement (IRD), UR-024, 911, avenue Agropolis, BP 64501, 34394 Montpellier Cedex 5, France. Tel.: 33 (0)4 67416162 - E-mail: boussinesq@ird.fr
}

\section{THE LOA LOA-RELATED POST-IVERMECTIN SERIOUS ADVERSE EVENTS}

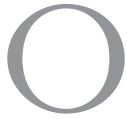
ne of the characteristics of Loa loa infection is that some individuals may harbour more than 30,000 microfilariae ( $\mathrm{mfs}$ ) per $\mathrm{ml}$ of blood. In these subjects, IVM may induce an encephalopathy which can be fatal (Boussinesq et al., 2003). These serious adverse events (SAEs), though rare, have a negative impact on the IVM therapeutic coverage in Loa-endemic areas and the risk of their occurrence precludes the launching of control programmes against lymphatic filariasis in central Africa. Two tools have been developed to identify loiasis-hyperendemic areas, where there is a risk of SAEs: a spatial model based on altitude and vegetation indices (Diggle et al., 2007), and a procedure for assessing the proportion of individuals with an history of "eye worm" (Takougang et al., 2002). Using these tools, it is now possible to delineate the areas where a enhanced monitoring should be applied to identify rapidly the SAE cases.

Besides this, assuming that the SAEs are due to the dramatic effect of IVM on Loa mfs, trials were conducted to identify a treatment that would have a more progressive effect than IVM, but would be efficient enough to reduce the high Loa loads below the risk threshold. By organizing mass treatments with such a regimen in Loa-endemic areas, it would be possible subsequently to distribute IVM with little risk of SAEs occurring. Unfortunately, none of the regimens tested so far, using albendazole, low doses of IVM, or antimalarials, met the desired objectives.

The third approach to tackle the problem aimed at clarifying the pathogenesis of the SAEs, to identify the most appropriate way to treat them. The lesions seen after IVM treatment in the brain of mandrills experimentally infected with Loa loa were consistent with thrombo-embolic disorders due to a massive mortality of $\mathrm{mfs}$ within the vessels. Studies are ongoing on a baboon model to assess the potential utility of some drugs in the early treatment of SAEs (Wanji S., personnal communication). 
Other researches are ongoing to identify co-factors that could play a role in the development of the SAEs, to evaluate whether the latter lead to neurological sequelae, and to assess the burden of loiasis.

\section{IS IT POSSIBLE TO STOP IVERMECTIN TREATMENTS, AND WHEN?}

$\mathrm{M}$ odelling studies suggest that IVM treatments alone would hardly lead to onchocerciasis elimination in Africa. However, as sustainable treatments is not warranted after cessation of APOC support, it is crucial to get the proof-of-principle whether elimination is feasible, and to define criteria to decide when IVM distribution can be stopped in an area. To do so, a study funded by the Gates Foundation is ongoing in West African foci where the parasitological and entomological indices of endemicity are very low. Treatments have been stopped in some foci and the populations are monitored to detect any recrudescence of infection. A related issue is the impact that repeated IVM treatments could have on the populations' immunity against Onchocerca volvulus and other pathogens. Results obtained in cattle infected with $O$. ocheng $i$ suggest that animals might be highly susceptible to infection after drug withdrawal (Njongmeta et al., 2004). Studies on this issue should be conducted in humans, taking into account the possible effects of coinfections (Mai et al., 2007).

\section{THE POSSIBLE EMERGENCE OF IVERMECTIN RESISTANCE IN O. VOLVULUS}

T VM is widely used since 1981 to control nematodes of animals. Since 1987, cases of IVM resistance have been reported in intestinal parasites of sheep, goats, cattle and horses. The question as to whether such a phenomenon can also occur in O. volvulus is all the more justified because IVM is used for $>20$ years against onchocerciasis with high therapeutic coverages, and because only a relatively small proportion of the parasite population is in refugia at the time of treatment. The main effects of IVM on O. volvulus are a microfilaricidal effect (leading to the destruction of skin $\mathrm{mfs}$ ) and an embryostatic effect (preventing the release of new $\mathrm{mfs}$ by the adult female worm) lasting for several months. In addition, IVM has a moderate macrofilaricidal effect and probably an effect on the $3^{\text {rd }}$ and $4^{\text {th }}$ stage larvae (L3 and L4). Thus, each of the parasite stages is submitted to a selection pressure by IVM. A phenotype of "low response" to IVM has been reported in patients treated repeatedly with IVM. In particular, a proportion of female worms collected from patients who had received at least nine doses showed "active" embryogrammes (with embryonic stages up to the $\mathrm{mfs}$ ) three months after the last treatment (Awadzi et al., 2004). It was also shown that the rate of repopulation of skin with mfs after treatment was higher than expected in villages treated repeatedly with IVM (OseiAtweneboana et al., 2007). These observations may indicate a reduced embryostatic effect of the drug in some patients. In addition, it has been shown that IVM induces a selection for the P-glycoprotein (PgP), P-glycoprotein-like protein (PLP) and $\beta$-tubulin genes in adult worms, and that a relationship exists between the genotype of the adult worms for the $\beta$-tubulin gene and their fertility (Prichard, 2007; Bourguinat et al., 2007). These results are a matter of concern, even if it has also been shown that IVM remains as efficient on $O$. volvulus $\mathrm{mfs}$ (the pathogenic stage) as it was 20 years ago. In fact, one of the main issues is that there is no consensus as to which indicator would reflect any changes in the embryostatic effect after repeated treatments. The rapidity with which the mf loads re-increase after a treatment might be not appropriate to assess such changes, and the way by which the embryogrammes can be used to do so is not clear. Thus, it is urgent to clarify what is a standard response of $O$. volvulus (particularly its adult stage) to IVM. The mechanisms by which IVM acts on mfs (which are relatively insensitive to IVM in vitro), and on adult worms (the mechanisms by which the release of $\mathrm{mfs}$ is prevented after treatment are not known), should also be clarified. Such researches would help to understand how IVM treatments induce genetic selections in O. volvulus, and to identify molecular markers to detect IVM-resistant populations.

\section{THE NEED FOR ALTERNATIVE DRUGS}

T The fact that IVM treatments will probably not lead to onchocerciasis elimination in Africa, and the risk of emergence of IVM-resistant parasites make it necessary to find alternative drugs (or drug combinations), preferably with macrofilaricidal or longlasting sterilizing effects. The modern approach to drug discovery is based on the identification and validation of protein targets followed by high throughput screening (HTS) using chemical libraries. However, the drugs which are presently the most promising for onchocerciasis control were identified using "empirical" processes. The discovery that most of the filarial species, including O. volvulus, contain Wolbachia endobacteria essential for parasite fertility and survival opened new perspectives for therapy. Following in vitro and preclinical studies showing that various antibiotics 
are effective against Wolbachia, clinical trials were conducted to evaluate the effects of doxycycline on O. volvulus. They demonstated that a treatment with $200 \mathrm{mg} /$ day of doxycycline for six weeks results in the sterilization, and the death of more than $60 \%$ of the adult female worms (Hoerauf et al., 2008). A study is under way in Cameroon to evaluate whether such a regimen is applicable using the community-directed strategy (Wanji S., personnal communication). However, the use of other antibiotics or strategies should also be considered. In vitro studies on O. gutturosa suggest that another tetracycline, minocycline, might have a higher macrofilaricidal effect than doxycycline (Townson et al., 2006). In addition, the Wolbachia genome could be exploited to identify more active drugs. Lastly, a study using the $O$. ocheng $i$-cattle model showed that oxytetracycline administered every month for six months eliminated $>60 \%$ of the adult worms (Gilbert et al., 2005). Such an intermittent regimen could be tested against $O$. volvulus.

The macrocyclic lactones are grouped into the avermectins (to which IVM belongs), and the milbemycins. Among the latter, moxidectin (MOX) is used for various veterinary indications. Examination of adult $O$. ochengi collected from cattle treated with MOX at monthly doses of 200 or $500 \mu \mathrm{g} / \mathrm{kg}$ showed that these regimens were not macrofilaricidal (Trees et al., 2000). However, probably due to its long elimination half-life, MOX brings about a much longer effect on O. ochengi mf loads than IVM. Following studies in healthy humans, a phase 2 trial was launched in 2006 by TDR, with the collaboration of Wyeth, the manufacturer of MOX, to compare the safety and efficacy of IVM and MOX against $O$. volvulus $\mathrm{mfs}$ and adult worms. Pending the results, decision as to whether a phase 3 trial should be conducted will be taken. Because of the similarity in the structure of IVM and MOX, it has been argued that IVM-resistant O. volvulus populations would also be resistant to MOX but this has to be proven. Lastly, the safety of MOX in patients infected with Loa should be assessed.

Cyclooctadepsipeptides are a group of anthelmintics discovered in the early 1990s. Among those, emodepside was found to be active against $\mathrm{mfs}$ and adult stages of Acanthocheilonema vitae and Litomosoides sigmondontis in a rodent model (Zahner et al., 2001). In vitro assays using $O$. gutturosa adult males and in vivo assays using the O. lienalis-mouse model showed that the drug is highly effective against Onchocerca sp. (Townson et al., 2005). Emodepside paralyses the body-wall and pharyngeal muscles of nematodes via a mechanism involving latrophilin-like receptors (Harder et al., 2005). As its mode of action is different from that of common anthelmintics, there is no risk of cross-resistance with the latter. The drug, combined with praziquantel, is already used in veterinary medicine. The results of a recent in vitro testing against $O$. volvulus adult worms, together with the animal toxicology data obtained by Bayer, its manufacturer, will allow to make a decision regarding further development of emodepside for human onchocerciasis (Hudson \& Nwaka, 2007).

Within the last years, the dynamics aimed at identifying and developing new drugs against tropical diseases has sped up considerably. This dynamics, which involves academic structures and pharmaceutical companies, has led to the launching, in 2006, of the TDR's Helminth Drug Initiative. Two approaches are used to discover anthelmintics. In the traditional biologically-driven selection, compounds are screened against intact worms. In contrast, the mechanism-based screening searches for compounds that selectively act on defined protein targets. The genome projects developed since 1994-1995 on Brugia malayi and O. volvulus have permitted (using the expressed sequence tags approach, knowledge of complete genome of Caenorhabditis elegans, and powerful bioinformatic tools), to identify $>4000$ genes in O. volvulus, to evaluate their levels of expression in each parasite stage, and to get information on potential targets for drugs and vaccines (Williams et al., 2002). The use of RNA interference (RNAi), which has revealed the function of thousands of genes in C. elegans, was expected to help in identifying gene products essential for the survival of filariae. However, it is difficult to extrapolate the findings obtained from C. elegans to other nematodes (Grant \& Behm, 2007). Besides this, application of RNAi to parasitic nematodes is difficult, though interesting results have been obtained in B. malayi and O. volvulus (Lustigman et al., 2004). Thus, it is important to develop research to analyse the RNAi machinery in parasitic nematodes and, as RNAi would miss gain-of function drug targets, to develop other functional genomics tools to validate new targets. Target prioritization for screening is also based on their specificity (assessed by comparative genomics), and their druggability, i.e. the possibility that they could be inhibited by small molecules (McCarter, 2004). The second condition necessary to identify new drugs by the mechanism-based approach is the availability of large numbers of compounds for which there is a good biological or biochemical rationale. Thanks to TDR, agreements have been ratified with major pharma for the supply of compound libraries, which can be optimized by combinatorial chemistry (Geary et al., 1999). The HTS technologies now permit to process millions of assays to test extremely low amounts of compounds against cloned recombinant targets. Lastly, HTS hits have then to be translated into in vitro and in vivo anthelmintic actives. With this respect, the assays to be used for screening efficiently potential micro- and/or macrofilaricides are being clarified (Townson et al., 2007). 


\section{VACCINE}

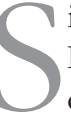
ince the mid 1980s, laboratories supported by the Edna McConnell Clark Foundation (EMCF) have conducted researches toward a vaccine against onchocerciasis. The proof-of principle for such a vaccine is provided by the existence of "putative immune" humans with no $\mathrm{mfs}$ despite constant exposure to infection, and by studies showing that animals immunized with irradiated Onchocerca sp. L3s were protected against infection challenges. Thus, the efforts focused on a vaccine based on antigens directed against the L3s or L4s, and not the mfs. The techniques mentioned above, as well as other approaches, have led to the identification and validation of a number of vaccine candidates (Lustigman et al., 2002). Studies were conducted in parallel to develop animal models to test the candidates and to define the mechanisms involved in parasite killing, and those by which the parasite subverts the host immune response. Besides the L. sigmondontis-mouse model, which is most useful for studying protective immunity (Allen et al., 2008), the models currently used for primary screen of vaccine candidates are the O. volvulus-mouse chamber model, and the $A$. vitae-jird life cycle model. Then the proteins found to be immunogens can be tested on the $O$. ochengi-cattle model. Though several proteins have been found to induce partial protection, it is clear that a vaccine against $O$. volvulus will require many years of development. More antigens need to be put in the animal testing pipeline and, besides proteins, other molecules should be examined as vaccine candidates.

\section{CONCLUSIONS}

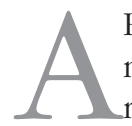

POC has to face a number of challenges, the most important being the increase in IVM coveCrage and in compliance, and the sustainability of treatments. However, more basic and operational research should also be conducted in the domain of biology. A global and coordinated approach, including all those involved in research on onchocerciasis should be encouraged. With this respect, the strategic plan for research developed to support the Global Program to Eliminate Lymphatic Filariasis constitutes a model. One of the main interests of the latter if that it aimed at clarifying some definitions. As mentioned above, similar needs exist for onchocerciasis (definition of criteria to stop IVM treatment, of what is a standard response to IVM, of what animal models to use, etc.). Despite the achievements of the onchocerciasis control programmes, it is urgent to address these issues proactively.

\section{ACKNOWLEDGEMENTS}

I thank Drs Catherine Bourguinat and Samuel Wanji for their comments on the manuscript.

\section{REFERENCES}

Allen J.E., Adjei O., Bain O., Hoerauf A., Hoffmann W.H., Makepeace B.L., Schulz-Key H., Tanya V.N., Trees A.J., WANJI S. \& TAYLOR D.W. Of mice, cattle, and humans: the immunology and treatment of river blindness. PloS Neglected Tropical Diseases, 2008, 2, e217.

Awadzi K., Boakye D.A., Edwards G., Opoku N.O., AtTah S.K., Osei-Atweneboana M.Y., Lazdins-Helds J.K., Ardrey A.E., Addy E.T., Quartey B.T., Ahmed K., Boatin B.A. \& Soumbey-Alley E.W. An investigation of persistent microfilaridermias despite multiple treatments with ivermectin, in two onchocerciasis-endemic foci in Ghana. Annals of Tropical Medicine and Parasitology, 2004, 98, 231-249.

Bourguinat C., Pion S.D.S., Kamgno J., Gardon J., Duke B.O.L., BoussinesQ M. \& Prichard R.K. Genetic selection of low fertile Onchocerca volvulus by ivermectin treatment. PLoS Neglected Tropical Diseases, 2007, 1, e72.

Boussinesq M., Gardon J., Gardon-Wendel N. \& Chippaux J.P. Clinical picture, epidemiology and outcome of Loa-associated serious adverse events related to mass treatment of onchocerciasis in Cameroon. Filaria Journal, 2003, 2 (Suppl. 1), S4.

Diggle P.J., Thomson M.C., Christensen O.F., Rowlingson B., Obsomer V., Gardon J., Wanji S., Takougang I., Enyong P., Kamgno J., Remme J.H., BoussinesQ M. \& Molyneux D.H. Spatial modelling and the prediction of Loa loa risk: decision making under uncertainty. Annals of Tropical Medicine and Parasitology, 2007, 101, 499-509.

Gilbert J., Nfon C.K., Makepeace B.L., Njongmeta L.M., Hastings I.M., Pfarr K.M., Renz A., Tanya V.N. \& Trees A.J. Antibiotic chemotherapy of onchocerciasis: in a bovine model, killing of adult parasites requires a sustained depletion of endosymbiotic bacteria (Wolbachia species). Journal of Infectious Diseases, 2005, 192, 1483-1493.

Grant W.N. \& Behm C.A. Target identification and validation for anthelmintic discovery. Expert Opinion on Drug Discovery, 2007, 2 (Suppl. 1), S91-S98.

Harder A., Holden-Dye L., Walker R. \& Wunderlich F. Mechanisms of action of emodepside. Parasitology Research, 2005, 97, S1-S10.

Hoerauf A., Specht S., Büttner M., Pfarr K., Mand S., Fimmers R., Marfo-Debrekyei Y., Konadu P., Debrah A.Y., Bandi C., Brattig N., Albers A., Larbi J., Batsa L., Adjei O. \& Büttner D.W. Wolbachia endobacteria depletion by doxycycline as antifilarial therapy has macrofilaricidal activity in onchocerciasis. A randomized placebo-controlled study. Medical Microbiology and Immunology, 2008, 197, 295-311.

Hudson A. \& Nwaka S. The concept paper on the Helminth Drug Initiative. Onchocerciasis/lymphatic filariasis and schistosomiasis: opportunities and challenges for the discovery of new drugs/diagnostics. Expert Opinion on Drug Discovery, 2007, 2 (Suppl. 1), S3-S7. 
Lustigman S., James E.R., Tawe W. \& Abraham D. Towards a recombinant antigen vaccine against Onchocerca volvulus. Trends in Parasitology, 2002, 18, 135-141.

Lustigman S., Zhang J., Liu J., Oksov Y. \& Hashmi S. RNA interference targeting cathepsin $\mathrm{L}$ and $\mathrm{Z}$-like cysteine proteases of Onchocerca volvulus confirmed their essential function during L3 molting. Molecular and Biochemical Parasito$\log y, 2004,138,165-170$.

Mai C.S., Hamm D.M., Banla M., Agossou A., Schulz-Key H., Heuschkel C. \& Soboslay P.T. Onchocerca volvulus specific antibody and cytokine responses in onchocerciasis patients after 16 years of repeated ivermectin therapy. Clinical and Experimental Immunology, 2007, 147, 504-512.

McCarter J.P. Genomic filtering: an approach to discovering novel antiparasitics. Trends in Parasitology, 2004, 20, 462468.

Njongmeta L.M., Nfon C.K., Gilbert J., Makepeace B.L., Tanya V.N. \& TREes A.J. Cattle protected from onchocerciasis by ivermectin are highly susceptible to infection after drug withdrawal. International Journal for Parasitology, 2004, 34, 1069-1074.

Osei-Atweneboana M.Y., Eng J.K.L., Boakye D.A., Gyapong J.O. \& PRIChard R.K. Prevalence and intensity of Onchocerca volvulus infection and efficacy of ivermectin in endemic communities in Ghana: a two-phase epidemiological study. Lancet, 2007, 369, 2021-2029.

PRICHARD R.K. Ivermectin resistance and overview of the Consortium for Anthelmintic Resistance SNPs. Expert Opinion on Drug Discovery, 2007, 2 (Suppl. 1), S41-S52.

Stolk W.A., Veerman L.J., De Vlas S.J. \& Habbema J.D. A rapid health impact assessment of the African Programme for Onchocerciasis Control (APOC). American Journal of Tropical Medicine and Hygiene, 2007, 77 (Suppl.), 103.

Takougang I., Meremikwu M., Wanji S., Yenshu E.V., Aripko B., Lamlenn S.B., Eka B.L., Enyong P., Meli J., Kale O. \& Remme J.H. Rapid assessment method for prevalence and intensity of Loa loa infection. Bulletin of the World Health Organization, 2002, 80, 852-858.

Townson S., Freeman A., Harris A. \& Harder A. Activity of the cyclooctadepsipeptide emodepside against Onchocerca gutturosa, Onchocerca lienalis and Brugia pahangi. American Journal of Tropical Medicine and Hygiene, 2005, 73 (Suppl.), 93.

Townson S., Ramirez B., Fakorede F., Mouries M.A. \& Nwaka S. Challenges in drug discovery for novel antifilarials. Expert Opinion on Drug Discovery, 2007, 2 (Suppl. 1), S63-S73.

Townson S., Tagboto S., McGarry H.F., Egerton G.L. \& TAYLOR M.J. Onchocerca parasites and Wolbachia endosymbionts: evaluation of a spectrum of antibiotic types for activity against Onchocerca gutturosa in vitro. Filaria Journal, 2006, 5, 4.

Trees A.J., Graham S.P., Renz A., Bianco A.E. \& Tanya V. Onchocerca ochengi infections in cattle as a model for human onchocerciasis: recent developments. Parasitology, 2000, 120, S133-S142.

Williams S.A., Laney S.J., Lizotte-Waniewski M., Bierwert L.A. \& UnNasch T.R. The River Blindness Genome Project. Trends in Parasitology, 2002, 18, 86-90.
Zahner H., Taubert A., Harder A. \& Samson-Himmelstjerna von G. Filaricidal efficacy of anthelmintically active cyclodepsipeptides. International Journal for Parasitology, 2001, 31, 1515-1522. 